\title{
Spontaneous Pneumothorax: A Complication of Coronavirus Disease 2019 (COVID-19) Patients
}

\author{
Qiannan Guo ${ }^{1}$ Peiwen Yang ${ }^{1}$ Kan-paatib Barnabo Nampoukime ${ }^{1}$ Ke Ma ${ }^{1}$ Haihao Wang ${ }^{1}$ \\ ${ }^{1}$ Tongji Hospital, Tongji Medical College, Huazhong University of \\ Science and Technology, Wuhan, Hubei, China \\ Thorac Cardiovasc Surg 2021;69:470-474.

\begin{abstract}
Address for correspondence Haihao Wang, MD, Tongji Hospital, Tongji Medical College, Huazhong University of Science and Technology, No. 1095 in Jiefang Avenue, Wuhan 430030, Hubei, China (e-mail: hhwang@tjh.tjmu.edu.cn).
\end{abstract}

\begin{abstract}
Keywords

- COVID-19

- spontaneous pneumothorax

- closed thoracic drainage

- poor prognosis

Background At present, the coronavirus disease 2019 (COVID-19) is spreading all over the world. The occurrence of spontaneous pneumothorax in these patients might be higher than the fact, and we should pay high clinical attention to them.

Method Data regarding clinical investigation, laboratory investigation, diagnosis, and treatment measures of 21 COVID-19 patients with spontaneous pneumothorax from January to March of 2020 were collected and analyzed in this study.

Results Seven patients had a history of basic lung diseases. All patients used different methods of oxygen therapy before the occurrence of spontaneous pneumothorax according to the severity of the COVID-19, including 18 patients with ventilator-assisted breathing, 2 patients with bilevel positive airway pressure assisted breathing, and 1 patient with mask oxygen inhalation. All patients were confirmed cases of COVID-19 by chest CT (computed tomography) and virus nucleic acid detection and were found to have spontaneous pneumothorax through physical examination, bedside X-ray, and/or bedside ultrasound. 13 of 21 patients combined with pleural effusion at the same time. All the patients underwent closed thoracic drainage for spontaneous pneumothorax and the pleural effusion, if any. Nine patients died, and 12 patients recovered smoothly.

Conclusion Spontaneous pneumothorax might be an overlooked complication of COVID-19 patients and may be associated with poor prognosis.
\end{abstract}

\section{Introduction}

Some cases of pneumonia with unknown reasons were first found in Wuhan, China, in December 2019, which were later identified to be caused by SARS-COV-2 (severe acute respiratory syndrome coronavirus 2 ) and is currently named by the World Health Organization as coronavirus disease 2019 (COVID-19). ${ }^{1}$ Until now, the COVID-19 has developed into a global disaster and needs global attention., ${ }^{2,3}$ In the clinical work of prevention and control of COVID-19, we found by accident that some patients are prone to acquire spontaneous pneumothorax, especially the severe and critical ones, and that these patients might be associated with adverse outcomes. This study mainly discusses the causes of spontaneous pneumothorax in COVID-19 patients and the treatment measures for them, so as to found the relation between

received

August 9, 2020

accepted after revision

September 22, 2020

published online

December 1, 2020

spontaneous pneumothorax and COVID-19 and to provide some clinical experience for saving the lives of these patients.

\section{Method}

In this study, the clinical information, past history, laboratory examination data, diagnosis, and treatment strategies of 21 COVID-19 patients with spontaneous pneumothorax, which occurred during hospitalization in Tongji hospital from January to March of 2020, were analyzed retrospectively. During this period, 207 cases including 108 (52.2\%) males and 99 (47.8\%) females were treated in our institution. In this study, the COVID-19 were diagnosed through clinical symptoms, epidemiological history, chest computed tomography (CT), and viral nucleic acid detection. The spontaneous pneumothorax was confirmed by clinical physical examination and/

(c) 2020. Thieme. All rights reserved.

Georg Thieme Verlag KG,

Rüdigerstraße 14,

70469 Stuttgart, Germany
DOI https://doi.org/ 10.1055/s-0040-1721061. ISSN 0171-6425. 
or imaging examination and mainly included chest radiograph and ultrasound at the bedside. All COVID-19 patients who suffered newly spontaneous pneumothorax during their treatment period regardless of whether they had a history of spontaneous pneumothorax or not were enrolled in the study. The Kircher method ${ }^{4}$ was used to calculate the compressed lung area of pneumothorax, and the detailed data are shown in $\boldsymbol{-}$ Table $\mathbf{1}$. The detailed clinical information, diagnosis, treatment strategies, and outcomes of these patients are shown in -Tables $\mathbf{1}$ and $\mathbf{2}$.

\section{Results}

Among the 21 patients included in the study, 18 (85.7\%) were male and 3 (14.3\%) were female, with an average age of $55.95 \pm 13.54$ years ( - Table 2 ). The proportion of patients over 60 years old accounted for $38.1 \%$ (- Table 2 ). Seven (33.3\%) patients had basic lung diseases, including three (14.3\%) cases of chronic obstructive pulmonary disease, one (4.8\%) with a history of pulmonary tuberculosis, one (4.8\%) with bronchiectasis, one (4.8\%) with previous pneumothorax, and one (4.8\%) with lung cancer (-Table 2). Of the 21 patients, 16 (76.2\%) patients had a history of smoking (-Table 2). Based on different degrees of hypoxia in patients, all patients received different forms of oxygen therapy, including 18 (85.7\%) cases of ventilator-assisted respiration, $2(9.5 \%)$ cases of bilevel positive airway pressure assisted respiration, and 1 (4.8\%) case of mask oxygen inhalation (-Table 2). The ventilator parameters of 18 patients with ventilator-assisted breathing are shown in - Table 2: all patients were accepted $\mathrm{FiO}_{2}$ (fraction of inspiration $\mathrm{O}_{2}$ ), which were more than $70 \%$ and positive end-expiratory pressure of these patients were between 0 and $3 \mathrm{~cm} \mathrm{H}_{2} \mathrm{O}$. The time between admission and pneumothorax ranged from 4 to 14 days, with an average of $8.24 \pm 3.40$ days ( - Table 2 ).

All patients were diagnosed with pneumothorax through clinical physical examination, bedside X-ray, and bedside ultrasound, with 12 (57.1\%) patients suffered unilateral pneumothorax and 9 (42.9\%) patients suffering bilateral pneumothorax, and 13 of $21(61.9 \%)$ patients combined with pleural effusion at the same time (-Table 1$)$. In all patients, bilateral pneumothorax occurred on one side first and on the other side later. Four (19\%) pneumothorax patients were complicated with mediastinal emphysema in the meantime, of which three were bilateral pneumothorax patients ( - Table 1 ). No subcutaneous emphysema was found in those patients. After definite diagnosis, all patients underwent unilateral or bilateral closed thoracic drainage to treat pneumothorax and pleural effusion, if any (-Table 1). In patients who underwent closed thoracic drainage, the bubbles in the chest bottle were monitored every day and bedside ultrasound was used to monitor the lung recruitment. When there was no bubble in the chest bottle and the lung recruitment was good, the chest tube was clamped for 24 hours. If there was no bubble recurrence by bedside ultrasound within 24 hours, the closed thoracic drainage tube could be removed. The specific extraction time in our study was shown in - Table $\mathbf{1}$. We considered that the best time to remove pleural effusion tube in patients with pleural effusion was when the pleural effusion was less than $50 \mathrm{~mL}$. The patients with pleural effusion in our study were all removed the tube within 3 days. According to the patient's dynamic condition, 6 (28.6\%) cases accepted extracorporeal membrane oxygenation (ECMO) to support their lives ( - Table 1). The duration of chest tube placement was 3 to 10 days (mean: $5.29 \pm 3.23$ days) ( - Table 1 ). Finally, 9 (42.9\%) patients died and $12(57.1 \%)$ patients recovered smoothly ( - Table 1 ). Among the nine death cases, 5 died of kidney failure, 3 died of respiratory failure, and 1 died of septicemia (-Table 1). In three COVID-19 patients with pneumothorax who died of respiratory failure, some tiny bubbles could be found in their lungs by CT scans on admission. Despite the positive measures were taken, the outcomes of these patients were still poor.

\section{Discussion}

As of March 11, 2020, 80,962 COVID-19 patients in China have been diagnosed and 3,162 have died, with the mortality rate being 3.9\%. ${ }^{5}$ In our study, the mortality rate of COVID-19 patients combined with spontaneous pneumothorax is as high as $42.9 \%$. Therefore, we suspect that spontaneous pneumothorax might be a sign of poor prognosis of COVID-19 patients, and we should pay high attention to these patients.

There is air between visceral pleura and parietal pleura, and the lungs contract continuously from the chest wall, which is called pneumothorax. ${ }^{6}$ From the clinical observation of the recent lung specimens obtained by autopsy of COVID-19 patients, a large amount of thick secretions can be seen on their lung surface, and the fibrous cord secretions secreted by infection were retained in the small airway, which damaged the ventilation and gas exchange function of lungs and blocked the small airway. As a consequence, the volume of dead cavity ventilation was increased and the pulmonary surfactant was decreased along with the decreased lung surface elasticity, which eventually can lead to the rupture of alveoli. Hyaline membrane was formed in the alveoli at the same time, which further hindered the exchange of oxygen and carbon dioxide between gas and blood. ${ }^{7-9}$ As the disease progresses, pulmonary fibrosis and consolidation may occur in these patients. ${ }^{9,10}$ These pathological changes suggest that the virus invades the lungs, impairs the lung function, and reduces the elasticity of the lungs after pulmonary fibrosis, which make pneumothorax more likely to occur. Positive airway pressure ventilation and other factors could further aggravate the pneumothorax. ${ }^{11}$ Similarly, in our study, some COVID-19 patients are more likely to develop pneumothorax, which may be related to decreased elasticity after pulmonary fibrosis and ventilatorrelated rupture of pulmonary bullae. In other words, the occurrence of pneumothorax is a complication and a sign of pulmonary dysfunction of COVID-19 patients.

In the current clinical work of three-level protection, auscultation cannot be effectively achieved; therefore, finding spontaneous pneumothorax in time has become a big problem. In our study, spontaneous pneumothorax was found in all patients by accident. Therefore, we suspected that spontaneous 

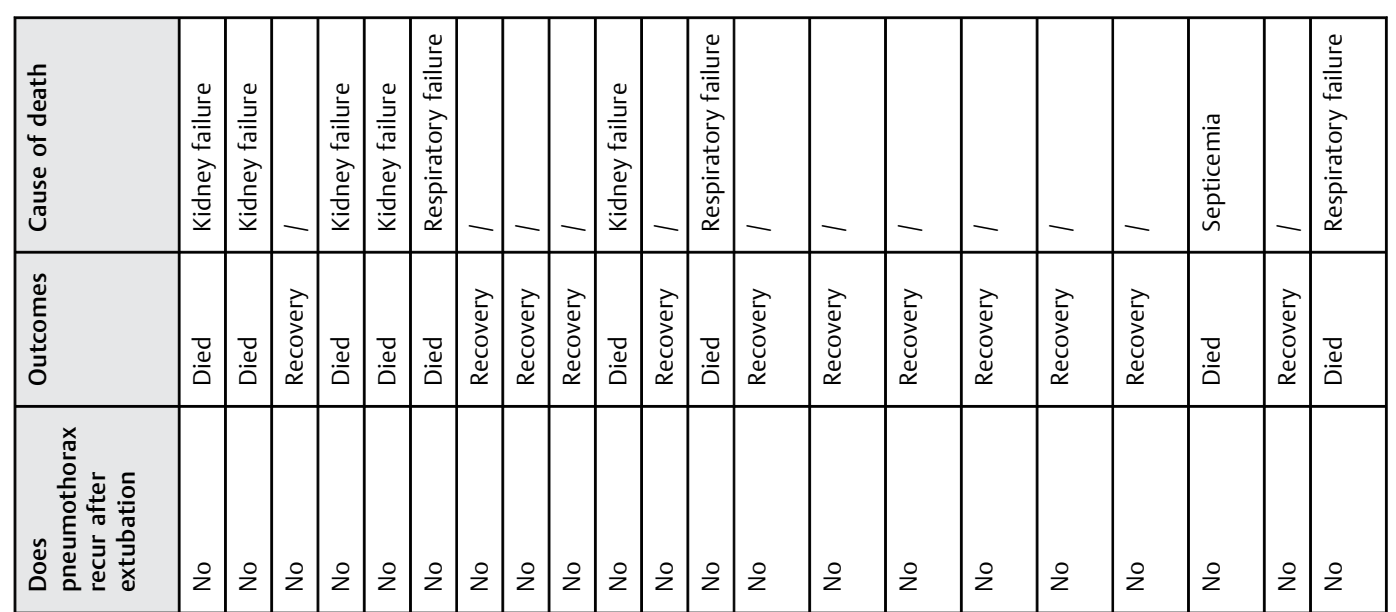

苟㐫

峞
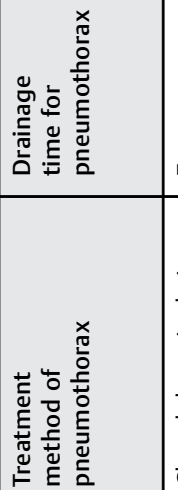

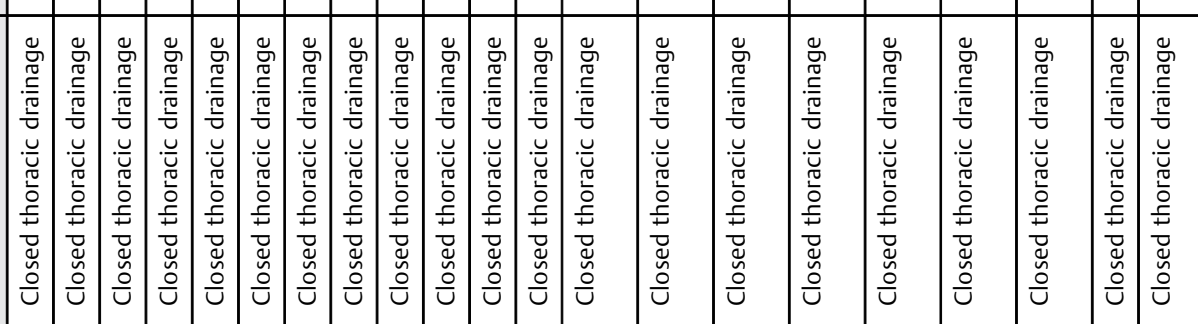

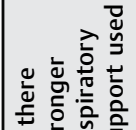

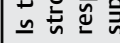

$\stackrel{\circ}{\sum}$

$\sum_{u}^{\circ} \sum_{u}^{\circ}$

$\sum_{u}^{\circ}-\sum_{u}^{\circ}$

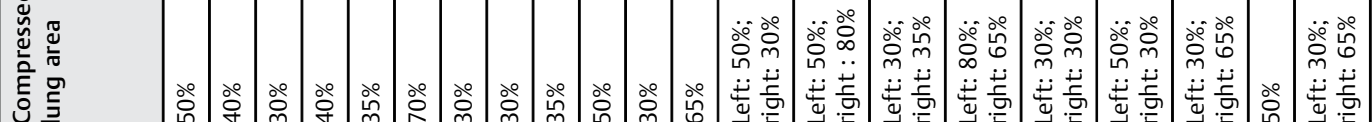

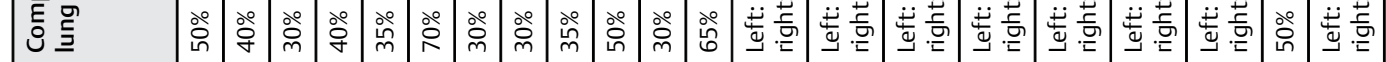

突

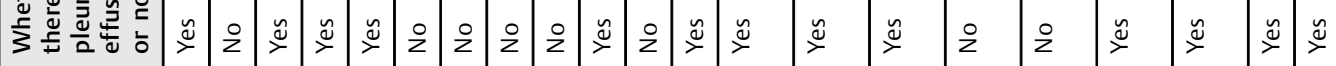

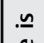

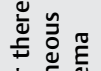

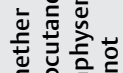

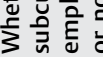

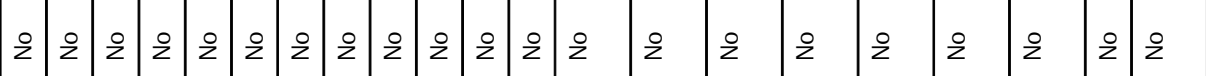

牙

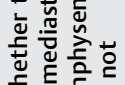

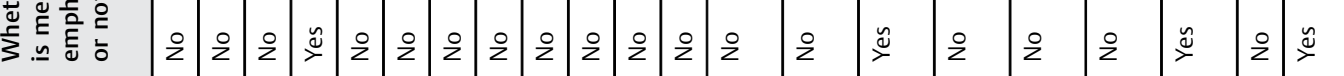

\begin{tabular}{|c|c|c|c|c|c|c|c|c|c|c|c|c|c|c|c|c|c|c|c|c|}
\hline 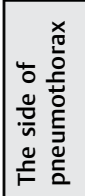 & $\begin{array}{l}\overrightarrow{\underline{E}} \\
. \overline{\underline{\alpha}}\end{array}$ & 苞 & 岃 & 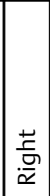 & $\mid \begin{array}{l}\vec{t} \\
\frac{\mathrm{g}}{\bar{\alpha}} \\
\end{array}$ & 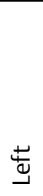 & 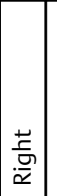 & $\begin{array}{l}\frac{\vec{v}}{.0} \\
\frac{.}{\alpha}\end{array}$ & 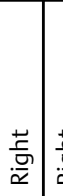 & 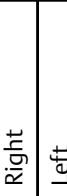 & 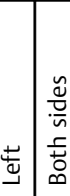 & 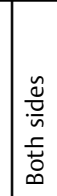 & 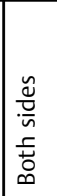 & 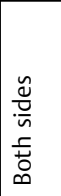 & 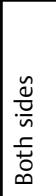 & 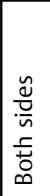 & 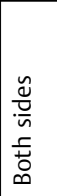 & $\begin{array}{l}\frac{\tilde{v}}{0} \\
\frac{\bar{n}}{n} \\
\text { 产 } \\
\infty\end{array}$ & 苞 & 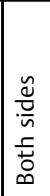 \\
\hline 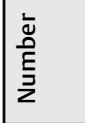 & - & $\sim$ & $m$ & $\nabla$ & ๓n & 0 & $\wedge$ & $\infty$ & $\sigma$ & $\circ 7$ & $=$ & $m$ & \pm & $\stackrel{\sim}{\circ}$ & $\stackrel{\varphi}{\circ}$ & $\Sigma$ & $\stackrel{\infty}{\longleftarrow}$ & $\stackrel{9}{9}$ & ㄱ & $\bar{N}$ \\
\hline
\end{tabular}




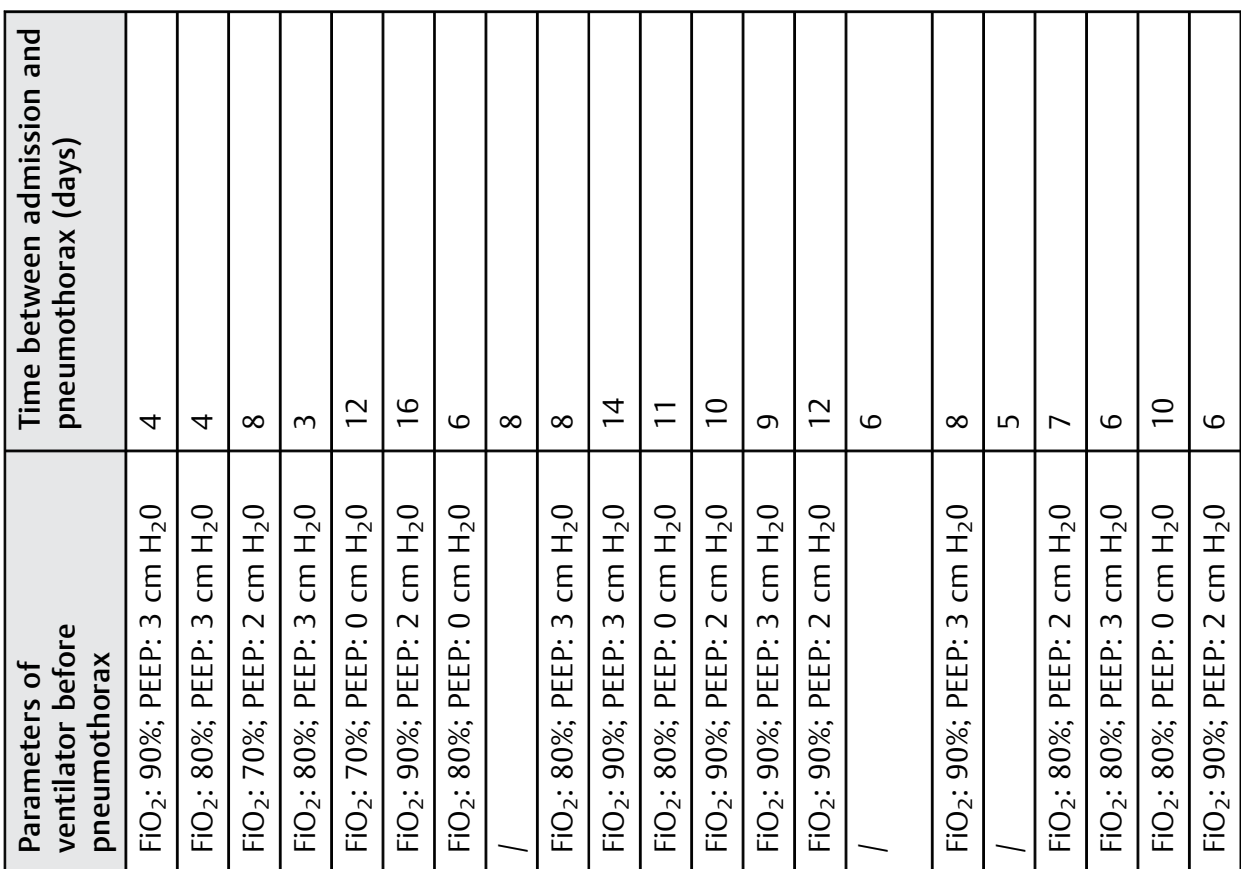

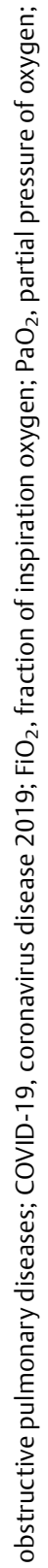

年

高

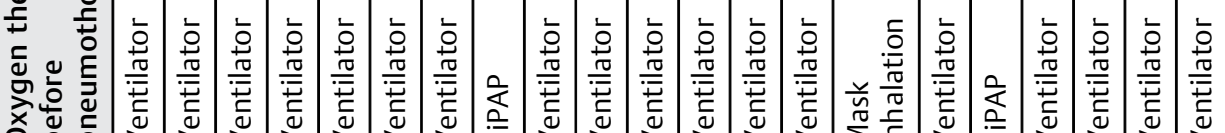

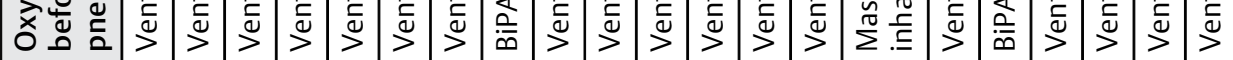

일

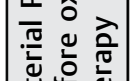

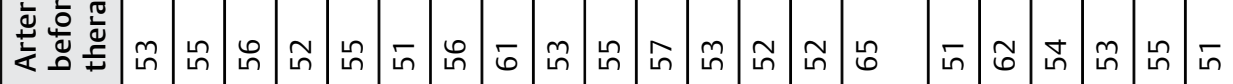

趇

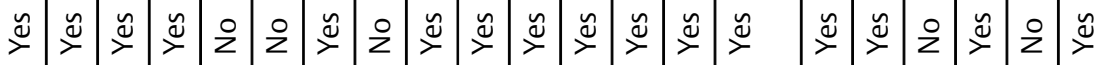

4

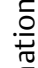

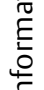

त

言

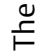

旁 (1)

年

$\int_{-}^{\infty} \frac{\infty}{\circ}{ }_{-}^{-}-$

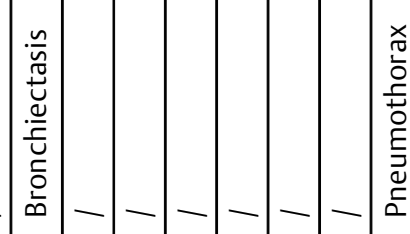

这

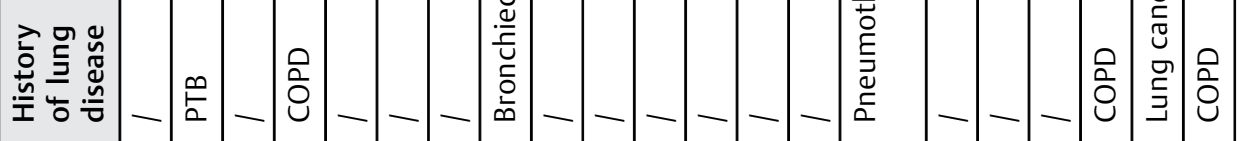

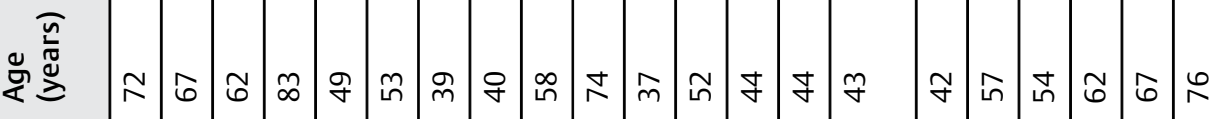

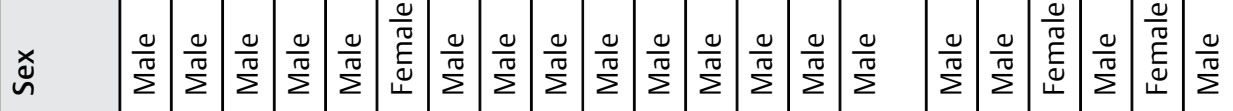

离

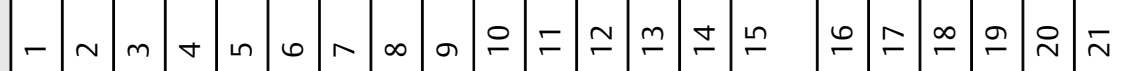

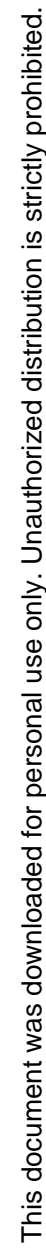
额

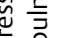


pneumothorax might be easily neglected in many COVID-19 patients especially those with mild symptoms. Three cases of pneumothorax were found in the autopsy of nine COVID-19 patients, but they are were diagnosed before death, indicating that the incidence of pneumothorax may be higher. ${ }^{12}$ Therefore, in our clinical work, we should pay close attention to the dynamic changes in patients' condition, find out the causes immediately, and take appropriate treatments as soon as possible to prevent the further deterioration of the condition.

In our clinical work, if the COVID-19 patients have sudden dyspnea accompanied by the decrease of blood oxygen saturation and the blood pressure, the increase of heart rate, and the decrease of unilateral and/or bilateral thoracic motion amplitude, the occurrence of pneumothorax should be highly suspected. ${ }^{13}$ At this time, we should take timely physical examination, bedside chest radiograph, and bedside ultrasound to make clear diagnosis. Bedside ultrasound may be more timely than bedside X-ray for severe patients due to its convenience as well as immediate availability of results, especially for patients with ventilator-assisted respiration after tracheal intubation. ${ }^{14-17}$

Clear diagnosis in time creates a good opportunity for early treatment of the patients. For the treatment of this kind of patients, the first thing is to perform thoracentesis or closed thoracic drainage timely according to the compressed area of the lungs. For severe or critical patients, especially whose respiratory function were assisted by ventilator and those with pleural effusion, closed thoracic drainage should be the primary choice because it can avoid repeated thoracentesis of the incompleteness of air extraction and the pneumothorax recurrence, and, in the meanwhile, it also can deal with the complication of pleural effusion if any. Conservative treatment can be used for a small amount of mediastinal emphysema, and all patients finally absorbed it by themselves. For the patients with unilateral pneumothorax, we also need to focus on the other side of the lung to prevent the missed diagnosis of contralateral pneumothorax or to discover the newly contralateral pneumothorax without delay. For patients with basic lung diseases, we need to strengthen the treatments of primary lung diseases simultaneously. For patients whose breathing function is not improved after active treatments, stronger respiratory support strategies may be needed, such as ECMO. ${ }^{18}$ During the treatment period of the patients, if their arterial blood oxygen index is improved, the ventilator parameters should be reduced slowly until the ventilator can be stopped. All patients in our study were cured after closed thoracic drainage, and there is no recurrence of pneumothorax during their hospitalization. For patients who are prone to the recurrence of pneumothorax or patients with giant pulmonary bullae, surgical treatment should be performed after the patients' condition is stable.

\section{Conclusion}

Active prevention and treatments of complications is an important part of the treatments of COVID-19 patients.
The occurrence of newly spontaneous pneumothorax might be a sign of disease deterioration of the COVID-19 patients. Therefore, for COVID-19 patients, especially in severe and critical cases, it is necessary to detect pneumothorax early and take active measures early in the meantime to prevent the patients from poor prognosis.

\section{Conflict of Interest}

None declared.

\section{References}

1 Marty AM, Jones MK. The novel coronavirus (SARS-CoV-2) is a one health issue. One Health 2020;9:100123

2 Cheng AC, Williamson DA. An outbreak of COVID-19 caused by a new coronavirus: what we know so far. Med J Aust 2020;212(09): 393-394.e1

3 Mahase E. Covid-19: UK records first death, as world's cases exceed 100 000. BMJ 2020;368:m943

4 Sayar A, Kök A, Citak N, Metin M, Büyükkale S, Gürses A. Size of pneumothorax can be a new indication for surgical treatment in primary spontaneous pneumothorax: a prospective study. Ann Thorac Cardiovasc Surg 2014;20(03):192-197

5 Coronavirus disease (COVID-2019) situation reports WHO. 2020; https://virusncov.com/coronavirus-cases-statistics

6 Swierzy M, Helmig M, Ismail M, Rückert J, Walles T, Neudecker J. Pneumothorax [in German]. Zentralbl Chir 2014;139(Suppl 1): S69-S86, quiz S87

7 Ramos C. Covid-19: the new pathology caused by a coronavirus [in Spanish]. Salud Publica Mex 2020;62(02):225-227

8 Tian S, Hu W, Niu L, Liu H, Xu H, Xiao SY. Pulmonary pathology of early-phase 2019 novel coronavirus (COVID-19) pneumonia in two patients with lung cancer. J Thorac Oncol 2020;15(05):700-704

9 Xu Z, Shi L, Wang Y, et al. Pathological findings of COVID-19 associated with acute respiratory distress syndrome. Lancet Respir Med 2020;8(04):420-422

10 Liu J, Zheng X, Tong $Q$ et al. Overlapping and discrete aspects of the pathology and pathogenesis of the emerging human pathogenic coronaviruses SARS-CoV, MERS-CoV, and 2019-nCoV.J Med Virol 2020;92(05):491-494

11 Schilling T, Kozian A. Which factors determine prognosis after ventilator-associated pneumothorax in mechanically ventilated patients? Minerva Anestesiol 2014;80(01):3-4

12 Bösmüller H, Traxler S, Bitzer M, et al. The evolution of pulmonary pathology in fatal COVID-19 disease: an autopsy study with clinical correlation. Virchows Arch 2020;477(03):349-357

13 Taylor G. Spontaneous pneumothorax. N Engl J Med 2000;343 (04):300, author reply 300-301

14 Espigares López MI, Meléndez Leal E, Pernia Romero A, Torres Morera LM. Pneumothorax: immediate ultrasound diagnosis in the critical patient [in Spanish]. Rev Esp Anestesiol Reanim 2018; 65(09):541-542

15 Hwang TS, Yoon YM, Jung DI, Yeon SC, Lee HC. Usefulness of transthoracic lung ultrasound for the diagnosis of mild pneumothorax. J Vet Sci 2018;19(05):660-666

16 Dahmarde H, Parooie F, Salarzaei M. Accuracy of ultrasound in diagnosis of pneumothorax: a comparison between neonates and adults-a systematic review and meta-analysis. Can Respir J 2019; 2019:5271982

17 Chan SS. Emergency bedside ultrasound to detect pneumothorax. Acad Emerg Med 2003;10(01):91-94

18 MacLaren G, Fisher D, Brodie D. Preparing for the most critically ill patients with COVID-19: the potential role of extracorporeal membrane oxygenation. JAMA 2020;323(13):1245-1246 